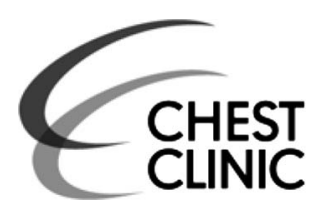
CLHEST

- Additional material is published online only. To view please visit the journal online (http://dx.doi.org/10.1136/ thoraxjnl-2015-207246)

${ }^{1}$ Department of Pediatrics, Hospital for Sick Children and University of Toronto, Toronto, Canada

${ }^{2}$ McMaster University,

Hamilton, Canada

${ }^{3}$ University of Manitoba,

Winnipeg, Canada

${ }^{4}$ University of Alberta,

Edmonton, Canada

${ }^{5}$ University of British Columbia,

Vancouver, Canada

${ }^{6}$ Environment Canada, Toronto, Canada

${ }^{7}$ University of Toronto, Toronto, Canada

${ }^{8}$ Northwestern University, Chicago, USA

${ }^{9}$ Simon Fraser University, Vancouver, Canada

\section{Correspondence to}

Dr Padmaja Subbarao

Department of Pediatrics, Hospital for Sick Children 555 University Avenue, Toronto ON, M5G 1X8, USA; padmaja.subbarao@sickkids.ca

Received 28 April 2015 Accepted 13 May 2015 Published Online First 11 June 2015

\section{CrossMark}

To cite: Subbarao $P$ Anand SS, Becker $A B$, et al. Thorax 2015;70:998-1000.

\title{
The Canadian Healthy Infant Longitudinal Development (CHILD) Study: examining developmental origins of allergy and asthma
}

\author{
Padmaja Subbarao, ${ }_{1}^{1}$ Sonia S Anand, ${ }^{2}$ Allan B Becker, ${ }^{3}$ A Dean Befus, ${ }^{4}$ \\ Michael Brauer, ${ }^{5}$ Jeffrey R Brook, ${ }^{6}$ Judah A Denburg, ${ }^{2}$ Kent T HayGlass, ${ }^{3}$ \\ Michael S Kobor, ${ }^{5}$ Tobias R Kollmann, ${ }^{5}$ Anita L Kozyrskyj, ${ }_{1}^{4}$ W Y Wendy Lou, ${ }_{1}^{7}$ \\ Piushkumar J Mandhane, ${ }^{4}$ Gregory E Miller, ${ }^{8}$ Theo J Moraes, ${ }^{1}$ Peter D Pare, ${ }^{5}$ \\ James A Scott, ${ }^{7}$ Tim K Takaro, ${ }^{9}$ Stuart E Turvey, ${ }^{5}$ Joanne M Duncan, ${ }^{2}$ \\ Diana L Lefebvre, ${ }^{2}$ Malcolm R Sears, ${ }^{2}$ the CHILD Study investigators
}

\section{ABSTRACT}

The Canadian Healthy Infant Longitudinal Development (CHILD) birth cohort study recruited 3624 pregnant women, most partners and 3542 eligible offspring. We hypothesise that early life physical and psychosocial environments, immunological, physiological, nutritional, hormonal and metabolic influences interact with genetics influencing allergic diseases, including asthma.

Environmental and biological sampling, innate and adaptive immune responses, gene expression, DNA methylation, gut microbiome and nutrition studies complement repeated environmental and clinical assessments to age 5 . This rich data set, linking prenatal and postnatal environments, diverse biological samples and rigorous phenotyping, will inform early developmental pathways to allergy, asthma and other chronic inflammatory diseases.

\section{INTRODUCTION}

Determining the origins of allergy and asthma has become an urgent research need. Developed countries face a high burden of allergic diseases and asthma, impacting families, healthcare systems and economies, while allergy and asthma are now also increasing in the developing world. Although family history of allergy or asthma is a risk factor, many children with asthma do not have a positive family history, suggesting that environmental influences are critical, acting either independently or by epigenetic mechanisms.

The Developmental Origins of Health and Disease $(\mathrm{DOHaD})$ hypothesis postulates that environmental exposures influence developmental pathways during critical periods of prenatal life and postnatal life, inducing permanent changes leading to altered disease susceptibility. Indoor and outdoor air pollutants, microbes, viral infections, maternal nutrition, infant feeding practices and psychosocial environments interact with genes and may exert their influence on asthma and allergy susceptibility via epigenetic mechanisms. The Canadian Healthy Infant Longitudinal Development (CHILD) Study is designed to address gaps in understanding complex gene-environment interactions during pregnancy and early childhood ${ }^{1}$ and provides a platform for study of atopic disease and other non-communicable diseases (NCDs) with early life origins.

\section{METHODS}

The cohort

Pregnant mothers in their second or third trimester were recruited from the general population in four communities across Canada (Vancouver, Edmonton, Manitoba (Winnipeg, Morden, Winkler) and Toronto) where local Research Ethics Boards approved the study. Inclusion and exclusion criteria for mothers and infants are in online supplementary table E1.

Two planned a priori substudies focused on psychological and infant and preschool lung function determinants and did not require full cohort participation.

\section{Assessments}

A timetable of assessments from pregnancy to 5 years is in online supplementary table E2.

Questionnaires (see online supplementary tables E2 and E3). Environmental, psychosocial, nutrition and health questionnaires are administered during pregnancy and at $3,6,12,18,24,30$ months, 3,4 and 5 years.

Environment. Household exposures are assessed repeatedly from recruitment to age 5 years. In addition to periodic questionnaires beginning at recruitment, a detailed walk-through assessment of the home was conducted when the child was 3-4 months old and included dust sampling for allergens and pollutants, along with collection of breast milk, child's urine, nasal swab and stool. Repeated biospecimens provide additional exposure biomarker information. Outdoor air pollution exposures are assessed using Land Use Regression models, residential history and time-activity patterns.

Psychosocial assessments. Maternal psychosocial characteristics assessed by self-report during pregnancy, post partum and at 1 year included socioeconomic status, stress, depressive symptoms and social relationships.

Nutrition. A Food Frequency Questionnaire (FFQ) is administered during pregnancy and at 
regular intervals for the child during the first 5 years.

Birth. The duration of labour, mode of delivery, anthropometrics, medication including antibiotics, and maternal or fetal complications were obtained from hospital records.

\section{Biological samples}

Blood samples. Whole blood, serum and plasma samples were obtained from all consenting mothers and fathers. ${ }^{2}$ Child blood was drawn at birth (cord blood), 1 year and 5 years. Cord blood from 785 infants has been prepared for studies of innate immunity and haemopoietic progenitor biomarkers.

Urine samples. Child urine was collected at 3 months, 1, 3 and 5 years, divided into aliquots, frozen at $-80^{\circ} \mathrm{C}$ and were then stored in liquid nitrogen.

Nasal samples. Nasal swabs (Copan Diagnostics, Corona, California, USA) were collected at 3 months and 1 year in all children, with additional swabs taken during acute illnesses in a subgroup, separated into aliquots, frozen at $-80^{\circ} \mathrm{C}$ and were then stored in liquid nitrogen.

Stool samples. Meconium was collected from infants at birth, and stool at 3 months and 1 year, divided into aliquots, frozen at $-80^{\circ} \mathrm{C}$ and were then stored in liquid nitrogen.

Breast milk. Ten millilitres of breast milk was collected at the home visit, divided into aliquots, frozen at $-80^{\circ} \mathrm{C}$ and then stored in liquid nitrogen.

\section{Clinic assessments}

At ages 1, 3 and 5 years, questions from the International Study of Asthma and Allergies in Childhood (ISAAC) were completed by the parent. At each assessment, the child is examined for evidence of atopic dermatitis, allergic rhinitis and asthma. Allergy skin prick tests and general anthropometrics are performed at all assessments. Blood pressure, waist circumference and skinfold thickness are measured at 3 and 5 years. Spirometry is performed in all children at age 5 years.

\section{Outcomes}

The primary outcome of the CHILD Study is expert physician diagnosed asthma at age 5 years (see online supplementary figure E1). Secondary outcomes include preschool wheezing, atopic sensitisation, atopic dermatitis, allergic rhinitis and food allergy. Permission was obtained to link with provincial administrative databases enabling studies of healthcare utilisation.

\section{RESULTS}

\section{Recruitment}

A total of 3624 families were recruited between 2008 and 2012. Gestational age at recruitment ranged from 6 to 39 weeks, mean 26.7 weeks and SD 6.3 (table 1, see online supplementary figure E2). Families who became ineligible after recruitment were excluded (miscarriage (15), prematurity $<35$ weeks (44), multiple births (5), pregnancy resulting from in vitro fertilisation (3) or complications, abnormalities or fetal death (15)) resulting in 3542 infants for the study. Study retention is $92 \%$ at age 1 year.

\section{Parents}

Mothers completed a health questionnaire during pregnancy $(n=3475)$, spirometry (3012), allergy skin testing (3073) and provided blood during pregnancy or at 1 year (3369). Of 2893 fathers recruited, 2841 have completed a health questionnaire, 2499 completed spirometry, 2519 underwent allergy skin testing and 2358 provided blood.

\section{Family demographics}

In over $25 \%$ of families, one or both parents were not of white Caucasian ethnicity (table 1). Just over half, 1868 of 3426 $(54.5 \%)$, of the index children were firstborns; the mean ages of mother and father were 32.3 and 33.8 years, respectively. Overall, 760 of 3424 mothers (22.2\%) and 547 of 3000 fathers (18.2\%) reported a personal history of asthma, 2832 of 3425 $(82.7 \%)$ and 2094 of $3000(69.7 \%)$, respectively, reported 'allergies' and 1768 of $3073(57.5 \%)$ and 1657 of 2432 $(68.1 \%)$ were objectively atopic by skin prick testing. Among mothers, 890 of 3229 (27.6\%) had ever smoked, while 185 of $3423(5.4 \%)$ reported smoking in pregnancy; 373 of 2847 $(13.1 \%)$ of fathers currently smoked. Significant differences observed among centres in housing characteristics have been described fully elsewhere. ${ }^{3}$

\section{Infant health outcomes}

The prevalence of caesarean section varied among centres (18.2-31.6\%, mean 25.6\%) (see online supplementary table E4). Cord blood was obtained in $75.1 \%$ of deliveries. The average birth weight was $3448 \mathrm{~g}$ (SD 482) and length $51.4 \mathrm{~cm}$ (SD 2.5); 52\% were males. Exclusive breast feeding at 3 months varied by site from $52 \%$ to $68 \%$. At 3 months, 1821 of 3111 infants $(58.5 \%)$ were exclusively breastfed as were 371 of 2679 (13.9\% of those with data) at 6 months.

\section{DISCUSSION}

Allergic diseases have reached epidemic proportions in middle-income to high-income countries, with a parallel increase in many chronic NCDs in the latter part of the 20th

Table 1 Baseline demographics of parents and families enrolled in the Canadian Healthy Infant Longitudinal Development (CHILD) Study by site

\begin{tabular}{lll}
\hline $\begin{array}{l}\text { Family demographics } \\
\text { Mean gestational age in weeks } \\
\text { at enrolment (SD) }\end{array}$ & $26.7(6.3)$ & \\
$\begin{array}{l}\text { Older siblings in the home (\%) } \\
\text { Parental demographics }\end{array}$ & 48.3 & \\
$\begin{array}{l}\text { Mean age in years (SD) } \\
\text { Reported ethnicity }\end{array}$ & $32.3(4.7)$ & Paternal \\
$\quad$ White Caucasian & $2532(72.9)$ & $2536(73.7)$ \\
$\quad$ South East Asian & $428(12.3)$ & $343(10.0)$ \\
South Asian & $91(2.6)$ & $106(3.1)$ \\
First Nations & $177(5.1)$ & $145(4.2)$ \\
$\quad$ Black & $77(2.2)$ & $110(3.2)$ \\
$\quad$ Other & $161(4.6)$ & $174(5.1)$ \\
$\quad$ Unknown & $6(0.2)$ & $29(0.8)$ \\
Education & & \\
$\quad$ High school or less & $295(8.7)$ & $502(14.7)$ \\
College or university & $2443(72.2)$ & $2151(63.1)$ \\
$\quad$ Postgraduate education & $647(19.1)$ & $756(22.2)$ \\
Health status & & \\
Reported asthma & $760 / 3424(22.2)$ & $547 / 3000(18.2)$ \\
Any reported allergy & $2832 / 3425(82.7)$ & $2094 / 3000(69.7)$ \\
Atopic by skin testing & $1768 / 3073(57.5)$ & $1657 / 2432(68.1) \dagger$ \\
Gestational diabetes & $115 / 3475(3.3)$ & $\mathrm{N} / \mathrm{A}$
\end{tabular}

Data are presented as $\mathrm{n}(\%)$ or as $\mathrm{n} / \mathrm{N}(\%)$ with $\mathrm{N}$ being the total sample available for that variable. Sample sizes differ for some characteristics due to differences in missing values.

*Those responding that they did not know their ethnicity are classified as 'Unknown'. tSkin test data apply only to participating fathers. 
century. In 2011, for only the second time in its history, the United Nations called a special General Assembly on health describing NCDs as a global epidemic. Asthma, one of the most common NCDs worldwide, is the earliest presenting chronic disease of childhood and the leading cause of childhood morbidity as measured by hospitalisations and school absences.

Lifestyle and environmental factors, including household and outdoor air pollution exposures, diet, physical activity and stress, appear to play important roles in the increase of these chronic diseases. Exposures occurring in utero and in infancy may be critical to the development of immune responses which influence the onset of these complex, chronic diseases, but how these exposures interact to promote allergic disorders versus immune homeostasis without clinical symptoms remains largely speculative.

This Canadian birth cohort study was initiated to study the development of allergy and asthma and designed with a focus on environmental assessments of infants and parents. The 3542 infants and families are predominantly from urban centres; over $80 \%$ of the Canadian population is urban. The recruited population is multicultural and ethnically varied, and children represent mixed ethnic populations to a greater degree than historical birth cohorts. The value of this cohort is enhanced by extensive phenotyping of both children and parents, characterisation of their environments and an extensive repository of biological samples. $^{2}$

A registry ${ }^{4}$ documented over 46 Canadian birth cohorts, comprising some 950000 individuals, of which CHILD is the only cohort focused on environmental effects on development of allergies and asthma in the general population. The National Institutes of Health and the European Commission research group 'Mechanisms of the Development of Allergy' (MeDALL) co-sponsored a workshop to harness knowledge generated across 130 birth cohorts initiated across many countries within the last 30 years, which have gathered data on asthma. ${ }^{5}$ However, few $(<12)$ encompass the breadth (eg, microbiome to psychosocial assessments) and depth (eg, infant lung function to innate immune phenotyping) of the CHILD cohort. We are actively participating in efforts to create standardised data definitions in conjunction with international initiatives, using standardised questionnaires and transparent data dictionaries to increase the power of such collaborations.

\section{CONCLUSIONS}

The CHILD cohort of 3542 eligible children followed to age 5 years with repeated surveys of environmental and other exposures, linking detailed prenatal and postnatal environments, a diversity of biological and environmental samples and careful assessment of developing clinical phenotypes will foster examination of relationships relevant to the development of allergy, asthma and other chronic NCDs with origins in childhood.
Acknowledgements We are grateful to all the families who are participating in this study, and the whole CHILD Study team, which includes interviewers, nurses, computer and laboratory technicians, clerical workers, research scientists, volunteers, managers and receptionists. Thanks to Chinthanie Ramasundarahettige for her careful work with data analysis and presentation. We also acknowledge the generosity of ALK-Abello, Mississauga, ON, Canada, in supplying all allergens for the study, and Lincoln Diagnostics Inc., Decatur, IL USA, for supplying the Duotip-Test II devices and skin testing kits.

Collaborators Scientific Advisory Committee: A Scientific Advisory Committee (Pau M O'Byrne (Chair), Fernando D Martinez, Mark E Raizenne, Felix A Ratjen, Peter D Sly, Erika von Mutius) provided valuable advice at start-up and has continued interacting with the Study leadership, contributing their experience in scientific studies, including longitudinal birth cohort studies in USA, Australia and Europe. CHILD Study investigators: Sears MR (Director), McMaster University; Subbarao P (co-Director), The Hospital for Sick Children; Allen R, Simon Fraser University; Anand SS, McMaster University; Becker AB, University of Manitoba; Befus AD, University of Alberta; Brauer M, University of British Columbia; Brook JR, University of Toronto; Chen E, Northwestern University, Chicago; Cyr M, McMaster University; Daley D, University of British Columbia; Dell S, The Hospital for Sick Children; Denburg JA, McMaster University; Elliott S, University of Waterloo; Grasemann H, The Hospital for Sick Children; HayGlass K, University of Manitoba; Hegele R, The Hospital for Sick Children; Holness DL, University of Toronto; Lou WYW, University of Toronto; Kobor MS, University of British Columbia; Kollmann TR, University of British Columbia; Kozyrskyj AL, University of Alberta; Laprise C, Université du Québec à Chicoutimi; Larché M, McMaster University; Macri J, McMaster University; Mandhane PM, University of Alberta; Miller G, Northwestern University, Chicago; Mogbel R (deceased), University of Manitoba; Moraes T, The Hospital for Sick Children; Paré PD, University of British Columbia; Ramsey C, University of Manitoba; Ratjen F, The Hospital for Sick Children; Sandford A, University of British Columbia; Scott JA, University of Toronto; Scott J, University of Toronto; Silverman F, University of Toronto; Takaro T, Simon Fraser University; Tang P, University of British Columbia; Tebbutt S, University of British Columbia; To T, The Hospital for Sick Children; Turvey SE, University of British Columbia.

Contributors This paper has been prepared by PS and MRS on behalf of the list of authors who have all contributed to its development and approved it for submission. PS and MRS serve as guarantors for the contents of this paper.

Funding The Canadian Institutes of Health Research (CIHR; AEC85761) and the Allergy, Genes and Environment (AllerGen) Network of Centres of Excellence provided core funding for the CHILD Study. Support has also been provided by Health Canada, Environment Canada, Canada Mortgage and Housing Corporation, the Sick Children's Hospital Foundation, Don \& Debbie Morrison, the Silver Thread Foundation and the Childhood Asthma Foundation.

Competing interests None declared.

Ethics approval Hamilton Regional Research Ethics Board.

Provenance and peer review Not commissioned; internally peer reviewed.

\section{REFERENCES}

1 Subbarao P, Becker A, Brook JR, et al. Epidemiology of asthma: risk factors for development. Expert Rev Clin Immunol 2009;5:77-95.

2 Moraes TJ, Lefebvre DL, Chooniedass R, et al. The Canadian Healthy Infant Longitudinal Development birth cohort study: biological samples and biobanking. Paediatr Perinat Epidemiol 2015;29:84-92.

3 Takaro TK, Scott JA, Allen RW, et al. The Canadian Healthy Infant Longitudinal Development (CHILD) birth cohort study: assessment of environmental exposures. J Expo Sci Environ Epidemiol. Published Online First: 25 Mar 2015. doi:10.1038/ jes.2015.7.

4 Joly MP, Boivin M, Junker $A$, et al. An inventory of Canadian pregnancy and birth cohort studies: research in progress. BMC Pregnancy Childbirth 2012;12:117.

5 Bousquet J, Gern JE, Martinez FD, et al. Birth cohorts in asthma and allergic diseases: report of a NIAID/NHLBI/MeDALL joint workshop. J Allergy Clin Immunol 2014; 133:1535-46. 